\title{
Study of antioxidant activity of pyrimidinium betaines by DPPH radical scavenging method
}

\begin{abstract}
The aim of this investigation was the study of the effect of some kinetic factors on the antioxidant activity of some synthesized pyrimidinium betaines, using 2, 2-diphenyl1-picrylhydrazyl (DPPH) free radical scavenging method. These betaines are bicyclics 1,2 and monocyclic 3 . We investigated the effect of concentration and reaction time on the antioxidant activity of these compounds and some standard antioxidants. All tested compounds were found to be active as DPPH scavengers but concentrations and reaction times influenced deeply the evaluation. At high concentrations, compound 3 is the best antioxidant compared to all other betaines. Reaction time is one of the most important criteria in this reaction. In comparison with standard antioxidants as ascorbic acid and the synthetic antioxidants butylated hydroxytoluene (BHT) and butylated hydroxyanisole (BHA), the data shows that, depending on the reaction time used, a different activity of the compounds is obtained: standard antioxidants react immediately with DPPH, while pyrimidinium betaines require longer reaction times and higher concentrations.
\end{abstract}

Keywords: antioxidant activity, concentration effect, DPPH, pyrimidinium betaine, radical scavenging, reaction time effect
Volume 8 Issue 2 - 2019

\section{Malki Fatiha, Touati Abdelkader \\ The Laboratory for Research on Bioactive Products and the Valorization of Biomass, Ecole Normale Supérieure,Algeria}

Correspondence: Malki Fatiha, The Laboratory for Research on Bioactive Products and the Valorization of Biomass, Ecole Normale Supérieure, 16308, BP 92,Vieux-Kouba str., Algiers, Algeria, Email Malki_fatiha76@yahoo.fr

Received: June 30, 2018 | Published: March 18, 2019

\section{Introduction}

Free radicals cause various human diseases. ${ }^{1}$ Antioxidants are substances that eliminate free radicals and serve as a defensive factor against their effects in the body. Antioxidants play an important role in the prevention of diseases induced by free radicals ${ }^{2}$ and systematically reduce their risk by reducing their concentrations. ${ }^{3}$ They act by inhibiting the initiation and propagation steps, resulting in the end of the reaction and delaying the oxidation process. ${ }^{4}$ In biological systems, antioxidants have multiple functions, eg it defends against oxidative damage by participating in the main cell signaling pathways and by preventing cell damage caused by the action of reactive oxygen species (ROS). Therefore, antioxidant consumption and the addition of antioxidant in food materials protect the body as well as food from deterioration of oxidation. ${ }^{5}$ Numerous methods to evaluate the antioxidant activity of specific compounds have been described, but the most widely documented relates to the 2, 2-diphenyl-1-picrylhydrazyl radical (DPPH) ${ }^{6-9} \mathrm{DPPH}$ is stable free radical that accepts an electron or hydrogen to become a stable diamagnetic molecule. In the DPPH radical-scavenging assay, antioxidants react with DPPH, and convert it to yellow- colored diphenylpicrylhydrazine. The color fading extent proves indirectly the radical-scavenging capacity of the antioxidant. ${ }^{6}$ The reduction of DPPH radical by antioxidants is evaluated by the decrease in absorbance at $517 \mathrm{~nm}$. The decrease in absorbance of DPPH radical caused by antioxidants is due to the reaction between antioxidant molecules and radical progress which results in the scavenging of the radical by hydrogen donation. ${ }^{10}$ The DPPH tests provided in the literature are based on the same principle as described by Brand- Williams et al., ${ }^{8}$ but the analytical protocols differ in several parameters. Evaluation of the methods and modifications for determination of the radical scavenging activity by DPPH shows that the main factors influenced the reproducibility are the solvent, duration of the reaction, sample to reagent ratio and the wave length for absorbance measurement of the decolouration of the reaction mixture. ${ }^{11}$ Therefore, it is obvious that these factors are of significance for the radical scavenging activity of the tested compounds.
Pyrimidinium betaines constitute a class of mesoionic compounds ${ }^{12}$ which have raised much interest as biologically active compounds. ${ }^{13}$ Pyrimidinium betaines remain an important class of bioactive heterocyclics due to the presence of the pyrimidine ring in their structure. The literatures indicated that compounds having pyrimidine nucleus have a broad range of biological activities. As a result of their remarkable pharmacological activity, intensive research on pyrimidine derivatives has been focused on their activity, thus constituting an integral part of pharmaceutical industry. ${ }^{14}$

In previous studies ${ }^{15,16}$ we have tested experimentally some synthesized pyrimidinium betaines through their ability to quench the synthetic 2,2- diphenyl-1-picrylhydrazyl (DPPH) radical. We have demonstrated in various in vitro assays ${ }^{16}$ that these compounds have antioxidative properties. In the present paper, the study of the reaction conditions for the determination of the activity of free radical scavenging by DPPH method was carried out. We investigated the effect of concentration and reaction time on the antioxidant activity of three synthesized pyrimidinium betaines and standard antioxidants, using 2,2-diphenyl-1-picrylhydrazyl (DPPH) free radical scavenging. These betaines are, bicyclics 1, 2 and monocyclic 3 (Figure 1).

\section{Material and methods}

\section{Material}

Chemicals and all solvents were of analytical grade and were purchased from Sigma-Aldrich, Merck, Prolabo and Biochem. In this study, pyrimidinium betaines 1-3 dissolved in ethanol were tested at concentrations $0-500 \mu \mathrm{g} / \mathrm{mL}$. Butylated hydroxyanisole (BHA), Butylated hydroxy toluene (BHT) and ascorbic acid (vitamin C) used as control standard antioxidants dissolved in ethanol were tested at concentrations $0-15 \mu \mathrm{g} / \mathrm{mL}$.

\section{Methods}

Synthesis of the compounds: The compounds including bicyclic pyrimidinium betaines: 1, 2 and monocyclic 3 were synthesized from amine derivatives and malonic esters by the reported procedures ${ }^{17-20}$ 
and their structures were determined by spectroscopic methods. These molecules have a common structure; they are mesoionic compounds. Their chemical structures are represented in Figure 1. Details of the synthesis and structural characterization of the compounds 1-3 can be found in Malki et al. ${ }^{19-21}$<smiles>[R2]c1c([O-])[n+]([R7])c2ccccn2c1=O</smiles><smiles>[R]c1c([O-])[n+]([R])c([R])n([R7])c1=O</smiles>

$I R_{1}=R_{2}=H \quad 3 R=R_{1}=R_{2}=C_{6} H_{5}$

$$
2 \mathrm{R}_{1}=\mathrm{H}, \mathrm{R}_{2}=\mathrm{C}_{6} \mathrm{H}_{5}
$$

Figure I Structures of studied compounds

DPPH radical scavenging assay: DPPH has been widely used for measurement of free radical scavenging ability of antioxidants. ${ }^{22}$ This method is based on the reduction of an alcoholic DPPH solution in the presence of a hydrogen-donating antioxidant. ${ }^{23}$ Hydrogen atom or electron-donation ability of the corresponding compounds were measured spectrophotometrically from the bleaching of the purplecolored methanol solution of 2,2-diphenyl-1-picrylhydrazyl (DPPH). ${ }^{24}$ In this study, antioxidant activity of tested compounds was measured using the stable radical 2, 2- diphenyl-1-picrylhydraziyl (DPPH). The free radical scavenging capacity of betaines was determined using the DPPH according to the method of Blois with some modifications. ${ }^{6} \mathrm{~A}$ solution of DPPH in methanol (0.004\%) was prepared and $1 \mathrm{~mL}$ of this solution was mixed with $1 \mathrm{~mL}$ of varying concentrations of betaines solution in ethanol. The reaction mixture was vortexed thoroughly and left in the dark at room temperature. The absorbance of the mixture was spectrophotometrically measured at $\lambda_{\max }=517 \mathrm{~nm}$ and compared to the standard antioxidants (BHT, BHA and ascorbic acid (vitamin C). DPPH radical scavenging activity (\% RSA) of compounds was calculated from the absorbance at the start $(0)$ and after some reaction time (T) according to the equation (1).

$$
(\% \text { RSA })=(\text { ABS-ATS }) / \text { ABS } \times 100
$$

Where ABS is the absorbance of blank sample (DPPH) solution without the compound to be tested and ATS is the absorbance of tested sample.

\section{Results and discussion}

\section{Determination of radical scavenging activity by DPPH method}

DPPH has been used extensively as a free radical to evaluate reducing substances ${ }^{25}$ and a reagent for investigating the free radical scavenging activities of compounds. ${ }^{26}$ As the electrons become paired off, the solution color faded stoichiometrically depending on the number of electron taken up. ${ }^{6}$ Hence, this assay provides an insight into the reactivity of the tested samples towards a stable free radical. ${ }^{27}$

In this study, the antioxidant scavenging activity of the tested compounds was studied by in vitro determination of DPPH scavenging capacity. ${ }^{15,16}$ and the effects of the concentration and the reaction time on their antioxidant activity were examined. Ascorbic acid, BHA and BHT were used as reference compounds.

\section{Effect of the concentration}

The results of DPPH radical scavenging rate (\%) of studied betaines $1-3$ at various concentrations at $\mathrm{T}=180 \mathrm{~min}$, are illustrated in Figure $2 \&$ Figure 3.

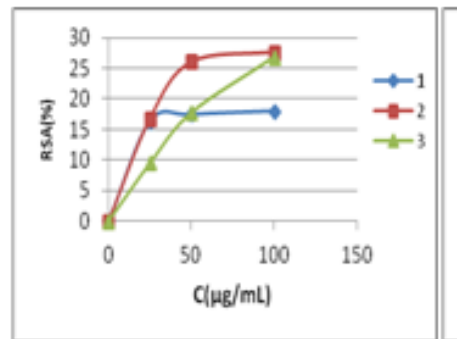

a) $\% \mathrm{RSA}, \mathrm{C}=100 \mu \mathrm{g} / \mathrm{mL}$,

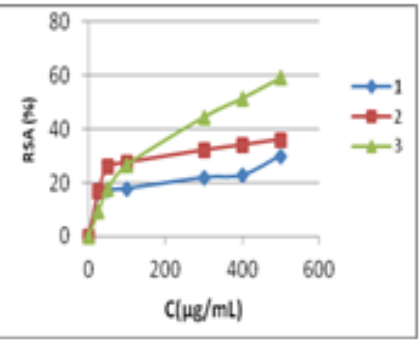

b) $\%$ RSA, $C_{\text {max }}=500 \mu \mathrm{g} / \mathrm{mL}$
Figure 2 Radical scavenging activity of tested betaines I-3.

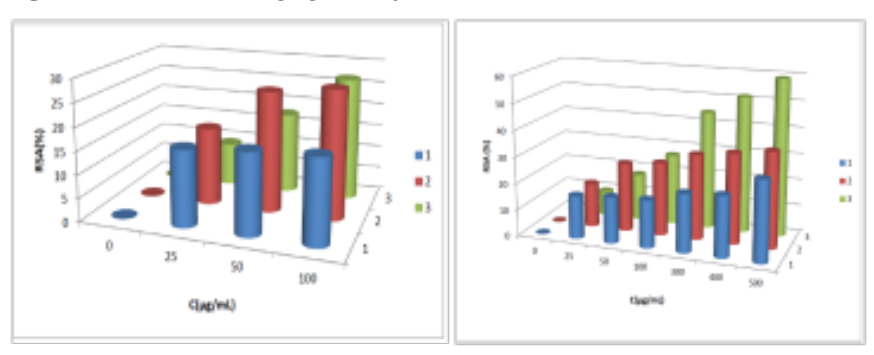

a) $\mathrm{C}=100 \mu \mathrm{g} / \mathrm{mL}$,

b) $C_{\text {max }}=500 \mu \mathrm{g} / \mathrm{mL}$

Figure 3 Antioxidant profile of tested betaines I-3.

From these figures, the DPPH radical scavenging activity towards pyrimidinium betaines is clearly demonstrated. As it can be seen, all tested betaines are active towards free radicals. The DPPH drop is concentration-dependent. The higher percentage of DPPH radical scavenging rate correlates with the higher concentrations. ${ }^{28}$ The decrease in the UV-visible absorbance of the DPPH radical engendered by tested betaines was due to the radical scavenging by electron donation. ${ }^{15}$ Results obtained in this study indicate different DPPH radical scavenging rate of tested compounds:

At lower concentrations (0- 25 $\mu \mathrm{g} / \mathrm{mL})$ (Figure 2a \& Figure 3a) the results show that, both bicyclic betaines 1and 2 exhibit the similar antioxidant activity that is higher than of monocyclic betaine 3. At $50 \mu \mathrm{g} / \mathrm{mL}$, bicyclic betaine 1 and monocyclic 3 have the similar activity $(17,73 \%$, and $18.01 \%)$ respectively, and at this concentration bicyclic betaine 2 is a stronger antioxidant $(26,04 \%)$ than previous betaines. At $100 \mu \mathrm{g} / \mathrm{mL}$ bicyclic betaine 2 has similar activity as monocyclic 3 $(27,67 \%, 26,82 \%)$ respectively.

At higher concentrations $(200-500 \mu \mathrm{g} / \mathrm{mL})$ (Figure 2b \& Figure $3 \mathrm{~b})$, monocyclic betaine $\mathbf{3}$ has the higher radical scavenging activity than both bicyclic betaines $\mathbf{1}$ and $\mathbf{2}$, and reaches the highest antioxidant activity at $500 \mu \mathrm{g} / \mathrm{mL}(59,20 \%)$ of DPPH radical scavenging rate, while bicyclics betaines $\mathbf{1}$ and $\mathbf{2}$ only $30,19 \%$ and 36,18\% respectively. The antioxidant activity of pyrimidinium betaines can be attributed to the conjugated systems involving nitrogen atoms which are known to stabilize free radicals. ${ }^{29}$ This conjugated systems are more extensive in monocyclic betaine $\mathbf{3}$ in comparison with the bicyclics $\mathbf{1}$ and $\mathbf{2}$. In $\mathbf{3}$ the radical formed is strongly stabilized by resonance through phenyl and carbonyl groups. ${ }^{16}$

On the basis of results obtained for all tested betaines, we concluded that at a low concentrations, bicyclic betaines show the highest DPPH radical scavenging rate, whereas at high concentrations monocyclic betaines show the highest DPPH radical scavenging rate.

The radical scavenging activity (\%RSA) of standard antioxidants (BHA, BHT and vitamin C) at concentrations $0-15 \mu \mathrm{g} / \mathrm{mL}(\mathrm{T}=180$ min) are shown in Figure 4. 

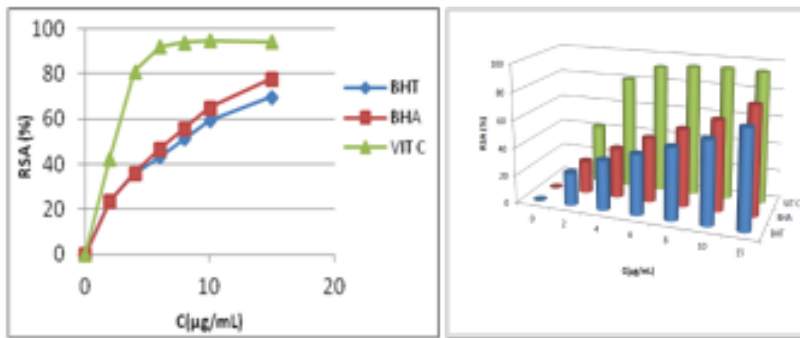

Figure 4 a) radical scavenging activity (\%RSA) of $\mathrm{BHA}, \mathrm{BHT}$ and vitamin $\mathrm{C}$ at concentrations $0-15 \mu \mathrm{g} / \mathrm{mL}$, b) antioxidant profile of $\mathrm{BHA}, \mathrm{BHT}$, vitamin $\mathrm{C}$ at concentrations $0-15 \mu \mathrm{g} / \mathrm{mL}$ )

The results indicate a significant decrease of DPPH radical due to the scavenging ability of tested standard antioxidants, and vitamin $\mathrm{C}$ is the most effective antioxidant. With regard to the molecular structures, the significant scavenging power of standards antioxidants was mainly due to the presence of hydroxyl groups in their structures on the phenyl ring which can donate hydrogen atom. ${ }^{30}$

Compared to the standard antioxidants (BHA, BHT and vitamin C), a lower activity of the tested betaines 1-3 was obtained. Indeed, at lower concentrations $(0-15 \mu \mathrm{g} / \mathrm{mL})$ standard antioxidants (BHA, BHT and vitamin C) exhibit the highest antioxidant activity (70-94\%) and betaines 1-3 (5-10\%). The low reactivity of pyrimidinium betaines towards DPPH radical can be attributed to the steric hindrance effects and the nature of the substituents on the hydrophilic head of betaine. ${ }^{16}$

We can conclude that scavenging activity differs between tested compounds. Since standard antioxidants increase the radical scavenging properties at a lower concentrations compared to the tested betaines.

\section{Effect of the reaction time}

Reaction time is an important criteria in the evaluation of the antioxidant activity.31 Indeed, all determinations of the DPPH radical scavenging activity reported in literature are based on the DPPH absorbance decrease very often measured at different reaction times: 30 min was used in the Blois method ${ }^{6}$ and shorter times $(20$, 10 and/or $5 \mathrm{~min}$ ) were reported by other authors. ${ }^{19-21}$ In this study, we followed kinetics' reaction of betaines 1-3, keeping constant their concentrations.

We studied the activity of the compounds' concentrations $(300 \mu \mathrm{g} /$ $\mathrm{mL}$ ) during $60 \mathrm{~min}$ and $180 \mathrm{~min}$. Figure 5 \& Figure 6 present the DPPH absorbance decrease as a function of time, in the presence of compounds 1-3 presented as (\%RSA), according to the equation (1). The data showed that, depending of the reaction time used, a specific activity of the compounds is obtained:

In the time interval 0-60 min: we obtained an almost same activity using bicyclic betaines 1 and 2, whereas monocyclic betaine 3 was more active (Figure 5a \& Figure 6a).

At maximal reaction time $T$ max $=180 \mathrm{~min}$, monocyclic betaine 3 reached its highest antioxidant activity $(44.57 \%)$. An activity of $32.02 \%$ and only $17.85 \%$ was obtained using bicyclic betaine 2 and 1 respectively (Figure 5b \& Figure 6b).

We concluded that the reaction time profoundly influenced the antioxidant activity of pyrimidinium betaines and that compound $\mathbf{3}$ is the best antioxidant compared to all other betaines.

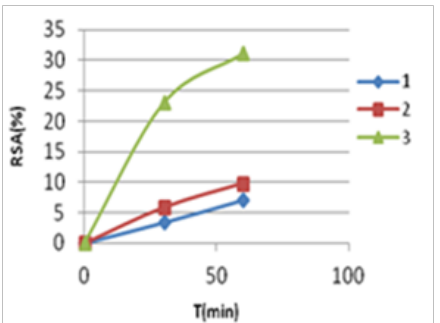

a) \%RSA, $T_{\max }=180 \mathrm{~min}$,

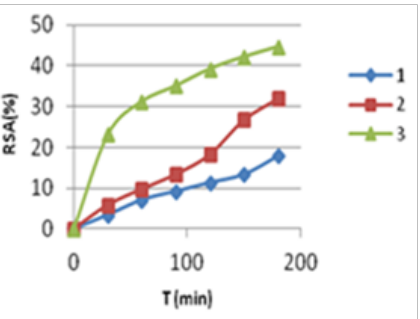

b) $\% R S A, T=60 \mathrm{~min}$
Figure 5 Radical scavenging activity of tested betaines I-3 as a function of time $(C=.300 \mu g / m L)$.

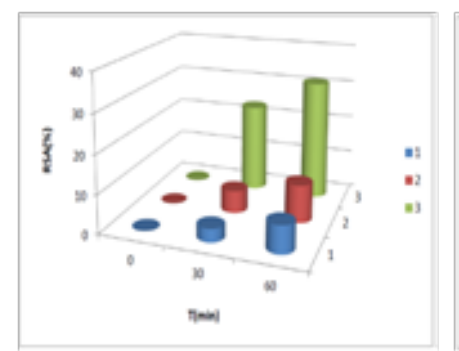

a) $\% R S A, T_{\text {max }}=180 \mathrm{~min}$,

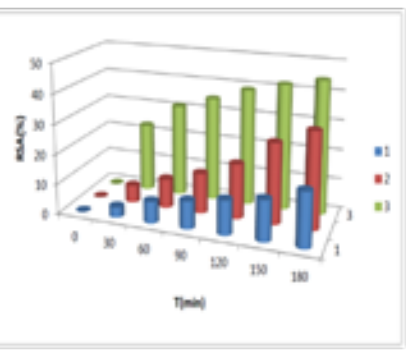

b) $\% R S A, T_{\text {max }}=60 \mathrm{~min}$
Figure 6 Antioxidant profile of tested betaines I-3 as a function of time $(\mathrm{C}=.300 \mu \mathrm{g} / \mathrm{mL})$.

\section{Conclusion}

The presented study indicates the antioxidant potential of synthesized pyrimidinium betaines. The results showed that at low concentrations bicyclic betaines $\mathbf{1}$ and $\mathbf{2}$ exhibit the highest DPPH radical scavenging whereas at high concentrations monocyclic betaine 3 reached the highest one. Among all tested betaines, compound 3 demonstrated strongest activity in all reaction times applied.

We can conclude that the reaction time and the concentration of the tested compounds are significant for their radical scavenging activity. Therefore, we can obtain much more information on the activity of the compounds by checking their reaction time and their concentrations.

\section{Acknowledgments}

None.

\section{Conflicts of interest}

The author declares that there is no conflicts of interest.

\section{References}

1. NJ Temple. Antioxidants and disease: more questions than answers. Nutrition Research. 2000;20:449-459.

2. P Thirunavukarasu, L Ramkumar, T Ramanathan, et al. Antioxidant activity of selected coastal medicinal plants. World Journal of Fish and Marine Sciences. 2010;2(2):134-137.

3. N Benkeblia, Free-Radical Scavenging Capacity and Antioxidant Properties of Some Selected Onions (Allium cepa L.) and Garlic (Allium sativum L.) Extracts. Brazilian Archives of Biology and Technology. 2005;48(5):753-759.

4. I Gulcin, V Mshvildadze, A Gepdiremen, et al. Antioxidant activity of a triterpenoid glycoside isolated from the berries of Hedera colchica: 3-O-(b-Dglucopyranosyl)- hederagenin. Phytother Res. 2006;20:130134 
5. S Palanisamy, S Sellappa, C Stella. Antioxidant properties of methanolic extract of Dictyota batresiana from south east coast of India, Journal of Pharmacy Research. 2010;3(12):2974-2976.

6. MS Blois. Antioxidant determinations by the use of stable free radical Nature. 1958;181:1199-1200.

7. P Molyneux. The use of the stable free radica diphenylpicrylhydrazyl (DPPH) for estimating antioxidant activity. Songklanakarin $\mathrm{J} \mathrm{Sci}$ Technol. 2004;26:211-219.

8. W Brand-Williams, ME Cuvelier, C Berset. Use of a free radical method to evaluate antioxidant activity. LWT - Food Science and Technology 1995;28:25-30.

9. V Bondet, W Brand-Williams, C Berset. Kinteics and mechanisms of antioxidant activity using free radical method. LWT - Food Science and Technology. 1997;30:609-615.

10. R Nalini, R Anuradha. Phytochemical Screening and «In-Vitro» Antioxidant Activity of Ethanolic Flower Extracts of Punica granatu. Int J Pharm Sci Rev Res. 2015;30(1):353-360.

11. G Marinova, V Batchvarov. Evaluation of the Methods for Determination of the Free Radical Scavenging Activity by DPPH. Bulgarian Journal of Agricultural Science, Agricultural Academy. 2011;17(1):11-24.

12. A Koch, U Jonas, H Ritterb, et al. Extended mesoionic systems: synthesis and characterization of monocyclic, polycyclic and macrocyclic pyrimidinium-olate derivatives and their photochemical behavior. Tetrahedron. 2004;60:10011-10018.

13. A Schmidt, $\mathrm{S}$ Wiechmann, $\mathrm{T}$ Freese. Recent advances in neutral and anionic $\mathrm{N}$-heterocyclic carbene-betaine interconversions, characterization and applications, Reviews and Accounts. ARKIVOC. 2013:424-469.

14. SD Arikkatt, BV Mathew, J Joseph, et al. Pyrimidine derivatives and it biological potential - A review. Int J Org Bioorg Chem. 22014;4(1):1-5.

15. F Malki, A. Touati and S. Moulay, Antioxidant Activity of Two Mesomeric Heterocyclic Betaines Containing a Pyrimidine Moiety. Pertanika J Trop Agric Sci. 2013;36(4):393-402.

16. F Malki, A Touati, S Moulay, et al. Evaluation of Antioxidant Activity of Some Mesoionic Pyrimidinium Betaines by Three Different Methods. International Journal of Chemical Engineering and Applications. 2016;7(6):373-377.

17. AE Tschitschibabin. Formation of bicyclic derivatives of $\alpha$ aminopyridine. Ber Dtsch chem Ges. 1924;57:1168-1172.

18. P Dvortsak, G Resofszki, M Huhn, et al. Reactions of pentachlorophenyl esters of malonic acid derivatives-II. Preparation and investigation of pyrimidine betaines. Tetrahedron. 1976;32:2117-2120.
19. F Malki, A Touati, S Rahal, et al. Total synthesis of monocyclic pyrimidinium betaines with fatty alkyl chain. Asian $J$ Chem. 2011;23:961-967.

20. F Malki, A Touati, S Moulay. Extraction and Recrystallization of Mesoionic Pyrimidinium Betaines. IJCEA. 2014;5 :151-154.

21. F Malki, A Touati, S Moulay. Use of Column Chromatography for Quantitative Isolation of Mesoionic Pyrimidinium Betaines. Journal of Research in Chemical, Metallurgical and Civil Engg. 2015;2(1):29-32.

22. J Pérez-Jiménez., S Arranz, M Tabernero, et al. Updated methodology to determine antioxidant capacity in plant foods, oils and beverages: extraction, measurement and expression of results. Food Research International. 2008;41(3):274-285.

23. İ Gülçin, E Kireçci, E Akkemik, et al. Antioxidant, antibacterial, and anticandidal activities of an aquatic plant: duckweed (Lemna minor L. Lemnaceae). Turk J Biol. 2010;34(2):175-188.

24. SY Çiftci, NG Kelekçi, US Gökşen, et al. Free-radical scavenging activities of 2- olated From Roots of Cassine TransvaalensisBurtt-Davy from Botswana. Afr J Biomed Res. 2008;11:55-63.

25. DMT Motlhanka, S Habtemariamc, PHoughton. Free Radical Scavenging Activity of CrudeExtracts and 4'-O-24. Methylepigallocatechin Is Organic and Bioorganic Chemistry. 2014;4(1):1-5.

26. XJ Duan, WW Zhang, XM Li. Evaluation of antioxidant property of extract obtained from a red alga. Polysiphonis ureceolta. Food Chemistry. 2006;95:35-43.

27. P Senthilkumar, S Sudha, C Stella. Antioxidant properties of methanolic extract of Dictyota batresiana from south east coast of India. J Pharm Res. 2010;3:2974-2976.

28. M Majewska, M Skrzycki, M Podsiad, et al. Evaluation of antioxidant potential of flavonoids: an in vitro study. Acta Pol Pharm. 2011;68(4):611615.

29. C Wentrup. The Neutral Reactive Intermediates in Organic Chemistry. New York: John Wiley and Sons;1984.

30. V Bondet, W Brand-Williams, C Berset. Kinetics and mechanisms of antioxidant activity using the DPPH free radical method. $L W T$ - Food Science and Technology. 1997;30:609-615.

31. VD Kancheva, Saso L, Angelova SE, et al. Antiradical and antioxidant activities of new bio-antioxidants. Biochimie. 2012;94:403-415. 